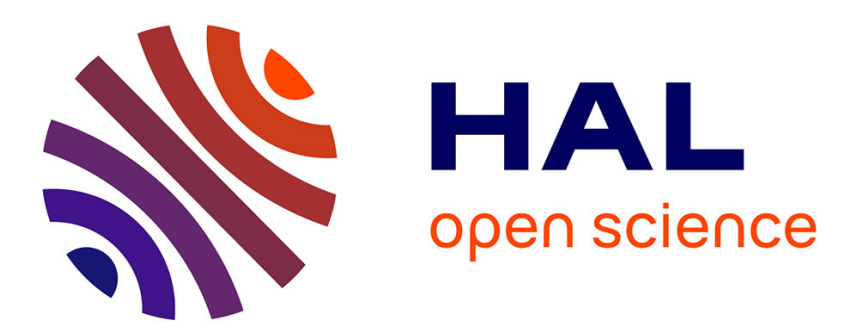

\title{
Collective infotaxis with reactive amoebae: a note on a simple bio-inspired mechanism
}

\author{
Nazim Fatès
}

\section{To cite this version:}

Nazim Fatès. Collective infotaxis with reactive amoebae: a note on a simple bio-inspired mechanism. 12th International Conference on Cellular Automata for Research and Industry, ACRI 2016, Sep 2016, Fez, Morocco. 10.1007/978-3-319-44365-2_15 . hal-01327983

\section{HAL Id: hal-01327983 \\ https://hal.inria.fr/hal-01327983}

Submitted on 7 Jun 2016

HAL is a multi-disciplinary open access archive for the deposit and dissemination of scientific research documents, whether they are published or not. The documents may come from teaching and research institutions in France or abroad, or from public or private research centers.
L'archive ouverte pluridisciplinaire HAL, est destinée au dépôt et à la diffusion de documents scientifiques de niveau recherche, publiés ou non, émanant des établissements d'enseignement et de recherche français ou étrangers, des laboratoires publics ou privés.

\section{(이) $\$$}

Distributed under a Creative Commons Attribution - NonCommercial - NoDerivatives $\mid 4.0$ 


\title{
Collective infotaxis with reactive amoebae: a note on a simple bio-inspired mechanism
}

\author{
Nazim Fatès \\ nazim.fates@loria.fr \\ Inria Nancy Grand-Est, LORIA UMR 7503 \\ F-54 600, Villers-lès-Nancy, France
}

June 7, 2016

\begin{abstract}
The purpose of this note is to study how to coordinate a team of agents that need to locate a hidden source on a two-dimensional discrete grid. The difficulty is that the source emits an information which can only be detected sporadically. This problem arises is various situations found in Nature, for instance when insects emit pheromones to attract their partners. A search mechanism named infotaxis was proposed to explain how agents may locate the source by using only intermittent detections.

Here, we study this problem in the context of stochastic cellular automata and reactive multi-agent systems. We present a bio-inspired model for the collective infotaxis search. The model, inspired by the behaviour of the social amoeba Dictyostelium, relies on the use of reaction-diffusion waves to guide the agents to the source. The formation of group is ensured by the non-simultaneous emissions of waves: the behaviour is totally decentralised and the agents successively act as emitters of waves or listeners, according to their local perceptions. We present a first study that shows that the model is worth of consideration and may provide a simple solution to the problem of coordinating a team to search a source of information with only sparse detections.
\end{abstract}




\section{Introduction}

The purpose of this note is to study how to coordinate a team of agents to perform a collective search in a two-dimensional environment. The objective of the team is to locate a source which emits an information that can only be detected sporadically. This problem arises in various situations studied in Biology, for instance when insects emit pheromones to attract their partners. There are cases where it is impossible to find the partner by following a gradient because the pheromones are dispersed in a turbulent environment: they are found in patches rather than with a smooth distribution. Vergassola et al. named infotaxis a search strategy which consists in maximizing the information gain on the location of the source 6 .

Here, we study this problem in the context of cellular automata: imagine a team of agents that are randomly dispersed on a two-dimensional grid and need to locate a particular cell, the emitting source. The agents have a very small memory; they have no map of their environment nor can they build one. They can only perceive the state of the cells situated at short distance. At each time step, the agents may detect the presence of the source (make a 'hit'), but without knowing the direction of the source, nor its distance. The detection probability increases as one gets closer the source and this is the only "hint" that can guide the agents. At each time step, the agents can move or change the state of the cell they are located on. Under such circumstances, how can a team of agents achieve a form of coordination in order to efficiently locate the source?

One may call this problem the distributed infotaxis problem with memoryless agents. In contrast, (classical) infotaxis has been mainly studied with agents that remember where the hits occurred. This allows them to build a map of their environment according to the detections they have made. Since the detections are stochastic events, the map is progressively adjusted. Interestingly, it was shown that a good search strategy does not necessarily consist in always trying to approach the source. In some cases, it may be more interesting to move orthogonally to the estimated direction of the source in order to improve the process of locating the direction of source [6. However, the use of a map in virtual agents or in real robots imposes a certain degree of complication. Different solutions have been proposed to simplify this scheme. In particular, Masson has proposed to use only a projection of the map of probabilities into a pre-determined form, where appears detection and non-detection terms [4]. Later on, this technique was extended by Zhang et al. to coordinate a team of robots [7].

We ask if we can simplify the solution further and if we can solve this problem with agents that do not remember where previous detections have occurred. In this context, the previous solutions cannot be used as the only thing that the agents can do is to use their respective positions on the grid to make better movements than they would do with a pure random walk.

The solution we propose is a variation of a previous model, the social amoebae [2], which was proposed to solve the decentralised aggregation problem: the 
agents need to gather and form a compact group in a totally decentralised way with only local perceptions and no memory of their previous actions. The aggregation scheme imitates the aggregation behaviour which occurs in the first phase of grouping in the social amoeba Dictyostelim discoideum. The mechanism heavily relies on the asynchronous emission of reaction-diffusion waves in the environment: each (virtual) amoebae needs to alternatively speak and listen and these different roles are governed by simple random events (see more details in Ref. [2]). If they all speak, (that is, emit waves) synchronously the aggregation process cannot occur.

We now modify this aggregation scheme to make the amoebae search for a hidden source. Informally, the system works as follows: each agent performs a random walk on the grid. When it makes a detection, which should be a rare event, it triggers a reaction-diffusion wave in the grid. This wave will propagate and attract the other agents towards the emitting agent. The agents that get closer to the source will have a tendency to emit more often that the agents that are far from it, and the group will collectively have a tendency to move closer to the source. Little by little, the group will progress towards the source and finally reach its target. The coordination of the group is ensured by the asynchronous nature of interactions: there are no leaders to take the decisions but instead each agent has the possibility to emit waves or to "listen" to the emissions of the others according to some particular stochastic behaviour that needs to be adjusted appropriately.

It can be noted that although seducing, the success of this scenario is not guaranteed. Indeed, this indirect communication process between agents, also called stigmergy, has to occur inside a group that is neither too compact nor too sparse. Indeed, an excessive grouping of agents is detrimental because the space they explore with their random movements is more limited than if the agents were absolutely free to wander on the grid. On the contrary, if the agents stay away from each other, this means that the attractive force of the reaction-diffusion waves is too weak to counter-balance the random movements of the agents. In this note, we explore this trade-off with a first set of numerical simulations. We show that the use of the virtual amoebae search scheme can be beneficial in some particular conditions. Since our experiments are limited, we will mainly aim at showing that this search strategy is worth of consideration. We do not bring any definitive answer to the problem of distributed infotaxis but rather ask questions that will need to be explored with a deeper analysis.

\section{Presentation of the model}

The space on which our model takes is defined a finite square grid denoted by $\mathcal{L}=\{1, \ldots, L\} \times\{1, \ldots, L\}$. Our model, the we could name the amobae-inf otaxis model, is composed of two interacting layers: the environment and the amoebae.

The environment is a cellular automaton composed of the cells of the grid. The state of the cells are taken in a set $Q$ that will be specified below. The global state of the environment is called a configuration and the set of configurations is 
denoted by $Q^{\mathcal{L}}$. At each time step, each cell calculates its new state according to its own state and the states of its neighbours and a possible influence from the amoebae. In other words, the environment is a cellular automaton which is in interaction with a system of reactive agents.

The emitting source is modelled by a distinguished cell, whose location $\sigma \in \mathcal{L}$ is fixed over the time. As mentioned earlier, in this problem, the cells can only make binary detections and the probability of detection decreases with the distance to the emitting source. This property is modelled by the introduction of a probability detection field $\rho: \mathcal{L} \rightarrow[0,1]$ which associates to each cell the probability to make a detection in this given cell. Here, we work with a field which follows an inverse square law ; it is described with: $\rho(\sigma)=1$ and

$$
\forall c \neq \sigma, \rho(c)=\frac{K}{d(c, \sigma)^{2}},
$$

where $d(x, y)=\left|x_{1}-y_{1}\right|+\left|x_{2}-y_{2}\right|$ is the Manhattan distance between two cells of the grid $x=\left(x_{1}, x_{2}\right)$ and $y=\left(y_{1}, y_{2}\right)$, and where $K<1$ is an arbitrary constant. Other authors have used an exponential decrease with the distance to the source. We prefer to use a $1 / d^{2}$ function in order to allow the existence of a small, but non neglible, probability of detection in the cells that are far from the source. Note that in our view, this field does not make the problem non-local: the probabilities are simply some fixed parameters that cannot be "read" directly by the cells or by the agents. Also note that this field may be computed by a pure cellular automaton if one wishes to do so.

The amoebae are reactive agents: they are described by their positions on the grid and their internal state, which is binary and taken in the set $I=\{\mathrm{rec}, \mathrm{emi}\}$ (emitter and receiver). For a set of $N$ amoebae, we will denote by $p=\left(p_{i}\right) \in \mathcal{L}^{N}$ the vector of all positions on the grid and by $s=\left(s_{i}\right) \in S^{N}$ the vector all internal states. The quantity $p_{i}$ and $s_{i}$ thus denote the position and the internal state of the $i$-th amoeba, respectively.

With these notations, we can describe our model with a dynamical system $F$, which operates on the space $Q^{\mathcal{L}} \times \mathcal{L}^{N} \times S^{N}$. For an initial condition $\left(x^{0}, p^{0}, s^{0}\right) \in Q^{\mathcal{L}} \times \mathcal{L}^{N} \times S^{N}$, its evolution is described by the recursive equation: $\left(x^{t+1}, p^{t+1}, s^{t+1}\right)=F\left(x^{t}, p^{t}, s^{t}\right)$. Let us now describe how these quantities are calculated 1

\section{Evolution of the cells}

The set of states of the cells is given by $Q=\{e, r, n\}$, which respectively correspond to the excited, refractory and neutral states. This environment implements a simple reaction-diffusion law, called the Greenberg-Hastings model(see e.g. Ref. [1]). Informally, a cell becomes excited if it is neutral and if it has at least one excited neighbour or if it is excited externally by an amoeba. An excited cell always becomes refractory and a refractory always become neutral.

\footnotetext{
${ }^{1}$ We make a slight abuse in notations because we use the formalism of classical dynamical systems even though our function $F$ is stochastic.
} 
To define this law, we introduce the function $S_{\mathbf{e}}$ that selects the excited cells around a given cell:

$$
S_{\mathbf{e}}(x, c)=\left\{d \in D, c+d \in \mathcal{L}, x_{c+d}=\mathrm{e}\right\}
$$

where $D=\{(0,1),(0,-1),(1,0),(-1,0)\}$ represents the four cardinal directions. This means that our cellular automaton uses the von Neumann neighbourhood but note that it can also be easily applied to other types of neighbourhoods [2].

We then define the modified Greenberg-Hastings law of evolution of the cells with, for all $c \in \mathcal{L}$ :

$$
x_{c}^{t+1}=\left\{\begin{array}{lr}
\mathrm{e} \text { if } x_{c}^{t}=\mathrm{n} \text { and } & \left(S_{\mathrm{e}}\left(x^{t}, c\right)\right. \text { is not empty } \\
\mathrm{r} \text { if } x_{c}^{t}=\mathrm{e} & \text { or } \left.\exists i, p_{i}^{t}=c \text { and } s_{i}^{t}=\mathrm{emi}\right) \\
\mathrm{n} \text { otherwise. } &
\end{array}\right.
$$

\section{Evolution of the amoebae}

First, we need to describe the fact that some amoebae will make a hit, that is, detect the source with a given probability which depends on their location. To this end we introduce the function $\mathrm{H}$, which, given a set of positions $p \in \mathcal{L}^{N}$, tells whether the $i$-th amoeba will make a 'hit':

$$
\forall p \in \mathcal{L}^{N}, \forall i \in\{1, \ldots, N\}, \mathrm{H}(p, i)= \begin{cases}1 & \text { with probability } \rho\left(p_{i}\right) \\ 0 & \text { with probability } 1-\rho\left(p_{i}\right) .\end{cases}
$$

The behaviour of the amoebae is as follows: at each time step, independently of the other amoebae, each amoeba may follow a random movement according to the four cardinal directions with probability $p_{\mathrm{A}}$, the probability of agitation. With probability $1-p_{\mathrm{A}}$, the "regular" behaviour is applied. This regular behaviour consists in the following rules:

1. if an amoeba is in the state rec, if it is standing on a neutral cell and sees one or several excited cells in its neighbourhood, it chooses one of these excited cells uniformly at random for its movement ; otherwise, if it has no excited neighbour, its stays on the same cell.

2. if an amoeba is in the state emi, if it is standing on a neutral cell, it turns this cell to the excited state. It then remains in state emi with probability $p_{\mathrm{R}}$, the probability of reemission.

The motivation for introducing the probability of agitation $p_{\mathrm{A}}$ is twofold: on the one hand, we add a randomness which ensures that the amoebae are not too static, on the other hand, this allows us to model the intrinsic noise that would exist in the movement of biological organisms or robots. The use of the probability of reemission $p_{\mathrm{R}}$ is not mandatory. It is simply here to allow several reaction-diffusion waves to be triggered when a hit occurs. As we will see below, 
these waves have an attractive force that groups the amoebae; this attractive force balances the dispersive force that is created by the random movements.

To write down this rule with our notations, we have:

- for $s_{i}^{t}=\mathrm{rec}, s_{i}^{t+1}= \begin{cases}\text { emi } & \text { if } \mathrm{H}\left(p^{t}, i\right)=1 \text { and } s_{i}^{t}=\mathrm{rec} \\ \mathrm{rec} & \text { otherwise }\end{cases}$

- for $s_{i}^{t}=$ emi, $s_{i}^{t+1}= \begin{cases}\text { emi } & \text { with proba. } p_{\mathrm{R}} \\ \text { rec } & \text { with proba. } 1-p_{\mathrm{R}}\end{cases}$

The positions evolve according to:

$$
p_{i}^{t+1}= \begin{cases}B\left[p_{i}^{t}, \mathcal{U}(D)\right] & \text { with proba. } p_{\mathrm{A}} \\ \mathcal{U}\left(S_{\mathbf{e}}\left(x^{t}, p_{i}^{t}\right)\right) & \text { with proba. } 1-p_{\mathrm{A}}\end{cases}
$$

where

- $\mathcal{U}(X)$ is a random variable that selects an element uniformly in a set $X$,

- $B[c, d]$ is a function that equals $c+d$ if $c+d$ is in $\mathcal{L}$ and $c$ otherwise,

- $S_{\mathrm{e}}$ is the set of excited cells neighbours to a given cell (see above).

The task is completed when an amoeba moves to the emitting source $\sigma$. The search time is the smallest $t$ which satisfies: $\exists i, p_{i}^{t}=\sigma$.

\section{First experiments}

Recall that our purpose is simply to test the relevance of doing a collective form of infotaxis with (almost) memoryless agents is feasible. For the sake of simplicity, we arbitrarily fix the number of amoebae to $N=20$ and the value of the constant in Eq. 1 to $K=0.5$. Similarly, we fix the reemission probability to $p_{\mathrm{R}}=0.9$ in order to allow for multiple waves to be emitted when a 'hit' occurs. A first visual experiment will be presented for $L=20$ and then statistics will be given for $L=80$.

\subsection{Qualitative observations}

In our experiments, the amoebae are placed uniformly at random on the grid. The emitting source is placed at coordinates $(L / 4, L / 4)$ in order to make the problem more difficult. Indeed, if it were placed at the centre, the amoebae could more easily find it since they do not need to displace the centre of gravity of the group.

First, let us observe visually how a system with only 5 amoebae behaves for a small grid of size $L=20$ and a small probability of agitation $p_{\mathrm{A}}=0.1$.

Figure 1 shows one particular random evolution of such a system. We see that various reaction-diffusion waves are emitted at different times when the hits occur. In this evolution two hits occur in a short time around $t=20$, which allows us to observe how the reaction-diffusion annihilate when they meet 


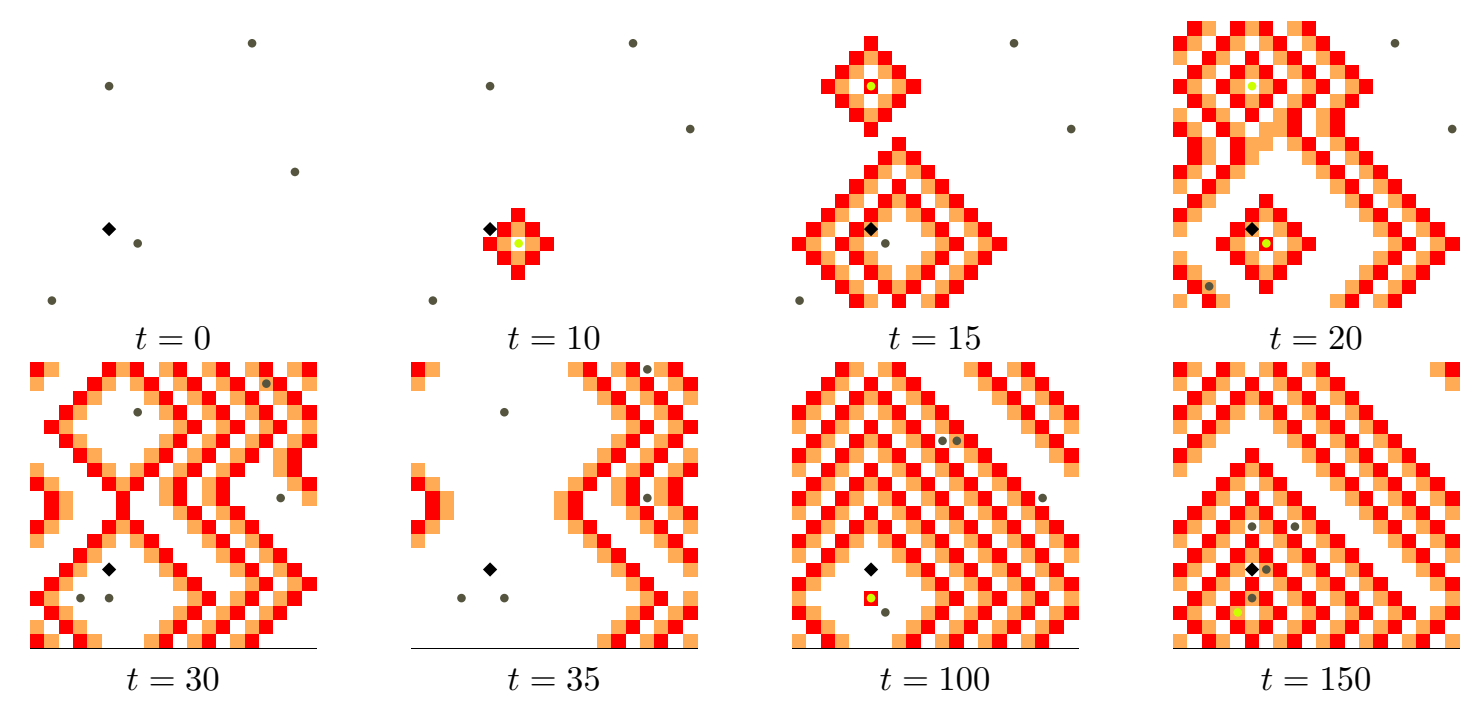

Figure 1: Evolution of the system for $N=5$ amoebae on a grid of size $L=20$ with $K=0.5, p_{\mathrm{A}}=0.1$, and $p_{\mathrm{R}}=0.9$; the emitting source is in black (diamond shape); amoebae are shown as circles in grey (rec state) or in yellow (emi state). The colors of the grid cells are red, orange or white, which respectively correspond to the e, $\mathrm{r}$ and $\mathrm{n}$ states. [color online]

frontally and how they merge when they meet orthogonally. This is the reason why it is important that the emission of waves do not occur simultaneously: as the reaction-diffusion waves annihilate when they meet, if all the amoebae were emitting at the same, none of them could receive the information. In other words, some amoebae need to remain in the rec state in order to be able to "feel" the waves emitted by the other amoebae. In this evolution, after a hundred steps the amoebae are already regrouped around the emitting source and at time $t=150$, we are very close to the source detection event.

\subsection{Quantitative experiments}

We now make a second step by considering the statistical variations of the search time as a function of the agitation probability $p_{\mathrm{A}}$. Indeed, $p_{\mathrm{A}}$ is the most important parameter in our study since it controls the average quantity of movement of the amoebae.

Taking the same settings as in the previous experiment, we observe the variation of the average detection time for a grid size of $L=80$. The agitation probability $p_{\mathrm{A}}$ varies from 0.1 to 1.0 with a step of 0.1 and the average search time is obtained with 1000 samples.

The results are shown on Fig. 2 and, interestingly, present a rather surprising behaviour of the system. It can be noted that a local minimum on the detection time is obtained for $p_{\mathrm{A}} \sim 0.2$. (An additional sampling point is added at 


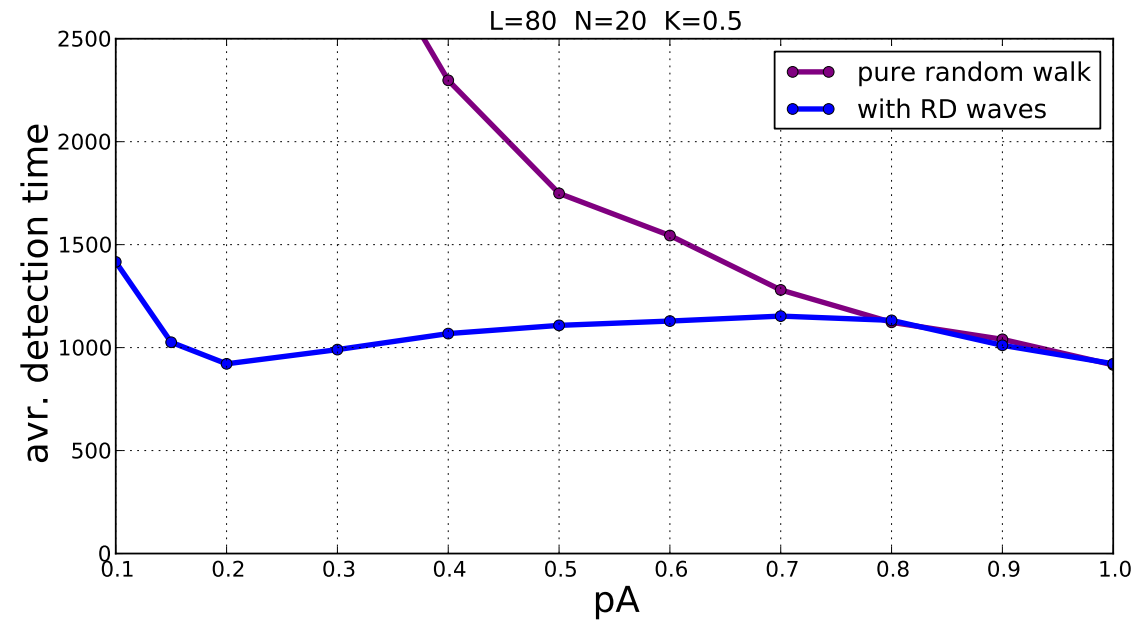

Figure 2: Search time for $N=20$ amoebae as a function of the agitation probability $p_{\mathrm{A}}$ for $L=80$ (in blue) with $K=0.5, p_{\mathrm{A}}=0.1$, and $p_{\mathrm{R}}=0.9$. The curve in purple shows the detection time when the amoebae follow a pure random walk (reaction-diffusion waves are suppressed).

$p_{\mathrm{A}}=0.15$ to have a better observation of this zone.)

We believe that the existence of this minimum corresponds to a balance between dispersive effect of the agitation and the grouping effect the reactiondiffusion waves. Indeed, if $p_{\mathrm{A}}$ is too small, the amoebae have a tendency to all aggregate on the same point - not the source! - and to all remain almost static close to this point. As the random movements are rare, if we imagine an amoeba that progressively gets far from the aggregation point, several emissions of waves emitted by the amoebae will call it back to this point. On the contrary, when $p_{\mathrm{A}}$ is too high, the disorder introduced by the agitation is no longer beneficial because the information gained by "listening" to the other amoebae is "diluted" by the random movements.

For values of $p_{\mathrm{A}}$ which are even larger, that is, greater than 0.75 , we observe that the detection time decreases again. This second effect is simply explained by considering the average number of movements per step: since the order of the system is already very weak (as $p_{\mathrm{A}}$ is high), it becomes more efficient to "mechanically" increase the average speed of the amoebae by moving more often.

In order to estimate what part of the behaviour is due to the reactiondiffusion waves and what part is due to the random movements, we estimated the average search time when there are no waves (we set $K=0$ ). The corresponding curve on Fig. 2 shows no surprise: it is decreasing with $p_{\mathrm{A}}$ and, more precisely, the average detection time varies as $1 / p_{\mathrm{A}}$. This is expected since the amoebae follow a pure Brownian motion and do not interact. We can observe that for small values of $p_{\mathrm{A}}$, the gain is important and for a similar average speed of 
movements, the search time with the amoebae-infotaxis search strategy may be divided a factor 5 with respect to the random walk strategy.

\section{Discussion}

This note presented a discrete model for performing a collective infotaxis task in an environment where only an intermittent information is available. The first experiments show that the use of reaction-diffusion waves is a simple means to perform a collective search of a hidden source. The mechanism we described relies on the probabilistic and asynchronous nature of the detections: since these events are rare, the agents of the system (the amoebae) can alternatively act as emitters or receivers and thus coordinate their movements to get closer and closer to the emitting source.

A continuation of the experimental work is needed to get more insights on how to use virtual amoebae to solve the decentralised infotaxis problem. Indeed, it is not clear yet in which situations it is more efficient to use this mechanism rather than a simple random walk. It is also important to get a more precise view on the scaling laws of the system: how does the search time depend on the number of amoebae and on the size of the grid? There are also questions which regard the possible improvement of the model. Here we presented a solution that uses only three states for the cellular automaton dynamics of the environment and only two states for the agents. The possibilities for further extending the behaviour of the agents are numerous, as for instance, making their emission auto-adjusting in order to have the correct balance between "listening" and "speaking".

In the context of applying these elements of research in the context of distributed robotics [3, 5, it would also be interesting to test the robustness of our scheme against several perturbations such as the introduction of obstacles in the grid or a non-regular distribution of the detection probabilities. 


\section{References}

[1] Hugues Berry and Nazim Fatès. Robustness of the critical behaviour in the stochastic Greenberg-Hastings cellular automaton model. International Journal of Unconventional Computing, 7(1-2):65-85, 2011. Available from: http://www.oldcitypublishing.com/IJUC/IJUCabstracts/ IJUC7 .1-2abstracts/I JUCv7n1-2p65-85Berry.html.

[2] Nazim Fatès. Solving the decentralised gathering problem with a reactiondiffusion-chemotaxis scheme - social amoebae as a source of inspiration. Swarm Intellligence, 4(2):91-115, 2010. doi:10.1007/s11721-010-0038-4.

[3] Nazim Fatès and Nikolaos Vlassopoulos. A robust scheme for aggregating quasi-blind robots in an active environment. International Journal of Swarm Intelligence Research, 3(3):66-80, 2012. doi:10.4018/jsir.2012070105.

[4] Jean-Baptiste Masson. Olfactory searches with limited space perception. Proceedings of the National Academy of Sciences, 110(28):11261-11266, 2013. doi:10.1073/pnas.1221091110

[5] Fernando Silva, Luís Correia, and Anders Lyhne Christensen. Modelling synchronisation in multirobot systems with cellular automata: Analysis of update methods and topology perturbations. In Ch. Georgios Sirakoulis and Andrew Adamatzky, editors, Robots and Lattice Automata, pages 267-293. Springer International Publishing, Cham, 2015. Available from: http://dx.doi.org/10.1007/978-3-319-10924-4_12, doi: 10.1007/978-3-319-10924-4_12.

[6] Massimo Vergassola, Emmanuel Villermaux, and Boris I. Shraiman. 'Infotaxis' as a strategy for searching without gradients. Nature, 445(7126):406409, 2007. doi:10.1038/nature05464

[7] Siqi Zhang, Dominique Martinez, and Jean-Baptiste Masson. Multi-robot searching with sparse binary cues and limited space perception. Frontiers in Robotics and AI, 2(12), 2015. doi:10.3389/frobt.2015.00012. 